Artigo

\title{
Modelagem Simplificada Para Estimativa do Balanço de Energia à Superfície em Escala Regional (R-SSEB)
}

\author{
Argemiro Lucena de Araújo ${ }^{1}$, Madson Tavares Silva ${ }^{1}$, Bernardo Barbosa da Silva ${ }^{1}$, \\ Carlos Antonio Costa dos Santos ${ }^{1}$, Marcos Renato Basílio do Amorim ${ }^{1}$ \\ ${ }^{1}$ Universidade Federal de Campina Grande, Unidade Acadêmica de Ciências Atmosféricas, \\ Campina Grande, PB, Brasil.
}

Recebido em 14 de Dezembro de 2015 - Aceito em 27 de Dezembro de 2016

\begin{abstract}
Resumo
Este estudo apresenta uma metodologia simplificada e ajustada a partir de algoritmos existentes a dados de sensores orbitais disponíveis para a região Nordeste do Brasil (NEB) com o intuito de operacionalizar a determinação espacial da evapotranspiração (ET) apenas com dados de sensoriamento remoto, utilizando dados MODIS/Terra do período de 2002 a 2011. Os resultados obtidos para diversos alvos espalhados pelo NEB foram comparados a dados CLM (Common Land Model) e MOD16. Obteve-se erro médio percentual (EMP) e erro médio absoluto (EMA) da ordem de 23,6\% e 181 mm.ano ${ }^{-1}$, respectivamente, nos valores de ET quando comparados a dados CLM. Avaliou-se a ET obtida pelo algoritmo proposto, com dados de ET do produto MOD16, o que resultou em valores de EMP e EMA de 38,6\% e $288 \mathrm{~mm}^{\text {.ano }}{ }^{-1}$, respectivamente. Os dados médios anuais da ET variaram de 482 a 1105 mm.ano ${ }^{-1}$ relativos a áreas de Caatinga e floresta tropical pluvial, respectivamente. Os resultados apresentaram-se condizentes com a literatura e evidenciaram que a metodologia proposta possibilita operacionalizar o cômputo da ET em toda a região Nordeste, não obstante a grande heterogeneidade topográfica e climática da região.
\end{abstract}

Palavras-chave: balanço de radiação, MODIS, calor latente, calor sensível, sensoriamento remoto, modelagem.

\section{R-SSEB: Simplified Modeling for Energy Balance Estimation to the Regional Scale Surface}

\begin{abstract}
This study presents a simplified methodology and adjusted from existing algorithms of orbital sensor data available for the Northeast region of Brazil (NEB) in order to operationalize the spatial determination of evapotranspiration (ET) only with remote sensing data, using Terra/MODIS data from 2002 to 2011. The results for various targets around the NEB were compared with CLM (Common Land Model) and MOD16 data. Was obtained average percentage error (MPE) and mean absolute error (MAE) of 23,6\% and 181 mm.year ${ }^{-1}$, respectively, when compared to CLM data. It was evaluated the ET obtained by the proposed algorithm with MOD16 ET data, resulting in EMP and EMA of 38,6\% and 288 mm.year ${ }^{-1}$, respectively. The average annual data from ET ranged 482-1105 mm.year ${ }^{-1}$ relating to areas of Caatinga and tropical rainforest, respectively. The results presented are consistent with the literature and showed that the proposed methodology enables operationalize the computation of ET throughout the Northeast, despite the large topographic heterogeneity and climate of the region.
\end{abstract}

Keywords: net radiation, MODIS, latent heat flux, sensible heat flux, remote sensing, modeling.

\section{Introdução}

A evapotranspiração (ET) é um fenômeno físico que transfere grandes volumes de água da superfície terrestre para a atmosfera através da evaporação da água do solo e da transpiração dos vegetais. A quantificação do consumo de água de grandes áreas e projetos de irrigação é importante para a utilização e manejo dos recursos hídricos e vem sendo cada vez mais aplicada em várias partes do planeta (Bastiaanssen et al., 2005; Silva e Bezerra, 2006; Allen et al., 2007b).

Autor de correspondência: Carlos Antonio Costa dos Santos, carlos.santos@ufcg.edu.br. 
A região Nordeste do Brasil (NEB) engloba uma grande área caracterizada climatologicamente como semiárida, onde a irregularidade espaço-temporal das chuvas e as altas taxas evaporativas provocam a escassez de água para a população e para sua agricultura. Nessa região, a produção para fins de agronegócio é quase que totalmente obtida através da irrigação e a maior parte da população sobrevive da agricultura de sequeiro. Tendo como objetivo a melhor utilização dos recursos hídricos da região, várias aplicações vêm sendo desenvolvidas ao longo do tempo na região para a estimativa da ET (Santos et al., 2007; Bezerra et al., 2008; Santos e Silva, 2008), do balanço de radiação (Silva et al., 2005; Di Pace et al., 2008), do cômputo dos fluxos de calor sensível e latente (Silva e Bezerra, 2006; Mendonça et al., 2012), da calibração de componentes do balanço de radiação (Araújo et al., 2012a) e do refinamento de imagens termais (Agam et al., 2007; Araújo et al., 2012b).

Basicamente, a ET pode ser obtida de forma pontual em experimentos espalhados por diversas regiões a partir de alguns dados de superfície, tais como o coeficiente de cultura, parâmetros climáticos, monitoramento da umidade do solo, medida dos fluxos de vapor, dentre outros. Contudo, essas estimativas pontuais não permitem a extrapolação espacial da ET em escala regional, o que é atualmente uma grande preocupação em numerosas práticas de gestão regional dos recursos hídricos e, em especial no manejo da irrigação local (Bastiaanssen et al., 1998a,b; Kite e Droogers; 2000; Schuurmans et al., 2003).

Com o desenvolvimento da tecnologia de satélites ambientais, a determinação das perdas de água de grandes áreas passou a ser obtida com grande facilidade e operacionalidade. Dentre os vários algoritmos desenvolvidos nos últimos anos, o SEBAL (Surface Energy Balance Algorithm for Land) (Bastiaanssen et al., 1998a) foi um dos que tiveram grande repercussão no cenário mundial e vem sendo aplicado em diversas partes do mundo (Bastiaanssen, 2000; Allen et al., 2005; Bezerra et al., 2008; Silva et al., 2012). Entretanto, novas técnicas para melhoria do SEBAL surgiram nos últimos anos. Podem-se destacar as correções propostas por Tasumi (2003), que sugeriu a implementação do aspecto/inclinação da superfície e calibração atmosférica para as bandas espectrais dos satélites, e Trezza (2002), que sugeriu a extrapolação da ET instantânea para valores diários, como as que proporcionaram os avanços necessários para o desenvolvimento do METRIC (Mapping Evapotranspiration at high Resolution with Internalized Calibration) (Allen et al., 2007a).

Um dos trabalhos pioneiros para estimativa da ET é o de Norman et al. (1995), que propõem um método de duas camadas para estimar com maior precisão os fluxos turbulentos e radiativos, separando-os em fluxos distintos de evaporação e transpiração. Entre outros trabalhos que surgiram nos últimos anos, destacam-se o de Roerink et al. (2000), que propuseram o Simplified Surface Energy Bal- ance Index (S-SEBI), pelo qual a fração evaporativa é calculada a partir de uma relação entre o albedo $(\alpha)$ e a temperatura da superfície (Ts). Su (2002) desenvolveu o Surface Energy Balance System (SEBS) para estimar a ET da superfície. Pela determinação de uma série de parâmetros físicos, o algoritmo estabelecido utiliza a rugosidade e a transferência de calor juntamente com características dinâmicas da superfície para produzir a fração evaporativa e, em seguida, conduzir à ET. Senay et al. (2007) propuseram o Simplified Surface Energy Balance (SSEB) para estimar a ET a partir de dados termais do MODIS e da evapotranspiração de referência global, este que foi aprimorado para um modelo operacional chamado SSEBop e apresentado em Senay et al. (2013). Além disso, Gao et al. (2011) apresentaram uma série de modificações para estimar a ET em áreas heterogêneas, que deram origem ao Surface Energy Balance with Topography Algorithm (SEBTA). Mu et al. (2007) propuseram um modelo para estimar a ET global pela combinação de dados meteorológicos e dados MODIS, que posteriormente foi melhorado em Mu et al. (2011). Teixeira et al. (2012) sugeriram o Simple Algorithm for Evapotranspiration Retrieving (SAFER) com o intuito de estimar a ET sem a necessidade de classificação da vegetação e de identificação de condições hidrológicas extremas.

Um grande problema que vem sendo discutido é a aplicação desses algoritmos em grande escala, como por exemplo, o NEB. Enquanto a solução do balanço de energia (BE) mostra-se bastante aplicável em diversas partes do mundo, os dados e requisitos necessários para obtenção dos vários termos da equação impedem uma aplicação operacional em grande escala (Senay et al., 2007). Atualmente no Brasil, a grande maioria das aplicações é destinada a projetos irrigados, que geralmente compreendem pequenas áreas, e a aplicações que necessitam apenas de medidas pontuais para estimativas dos fluxos devido à carência de modelos aplicáveis regionalmente. Outro fator que merece atenção é que a escassez de estações de superfície para obtenção dos dados necessários a vários algoritmos vem impedindo a implantação dos mesmos.

Neste sentido pretende-se empregar uma metodologia simplificada e ajustada a partir de algoritmos existentes a dados de sensores orbitais disponíveis para o NEB com o intuito de operacionalizar o $\mathrm{BE}$ em grande escala sem a necessidade de dados de superfície.

\section{Dados e Metodologia}

\section{1. Área de estudo}

A região Nordeste do Brasil (NEB) possui uma área de aproximadamente $1.558 .196 \mathrm{~km} 2$ e uma população de 53.081.510 habitantes (IBGE, 2010) (Fig. 1). A mesma está localizada entre os paralelos de $01^{\circ} 02^{\prime} 30^{\prime \prime}$ e $18^{\circ} 20^{\prime} 07^{\prime \prime} \mathrm{S}$ e entre os meridianos de $34^{\circ} 47^{\prime} 30^{\prime \prime}$ e $48^{\circ} 45^{\prime} 24^{\prime \prime}$ W. Limita-se a norte e a leste com o oceano Atlântico, ao sul com os 


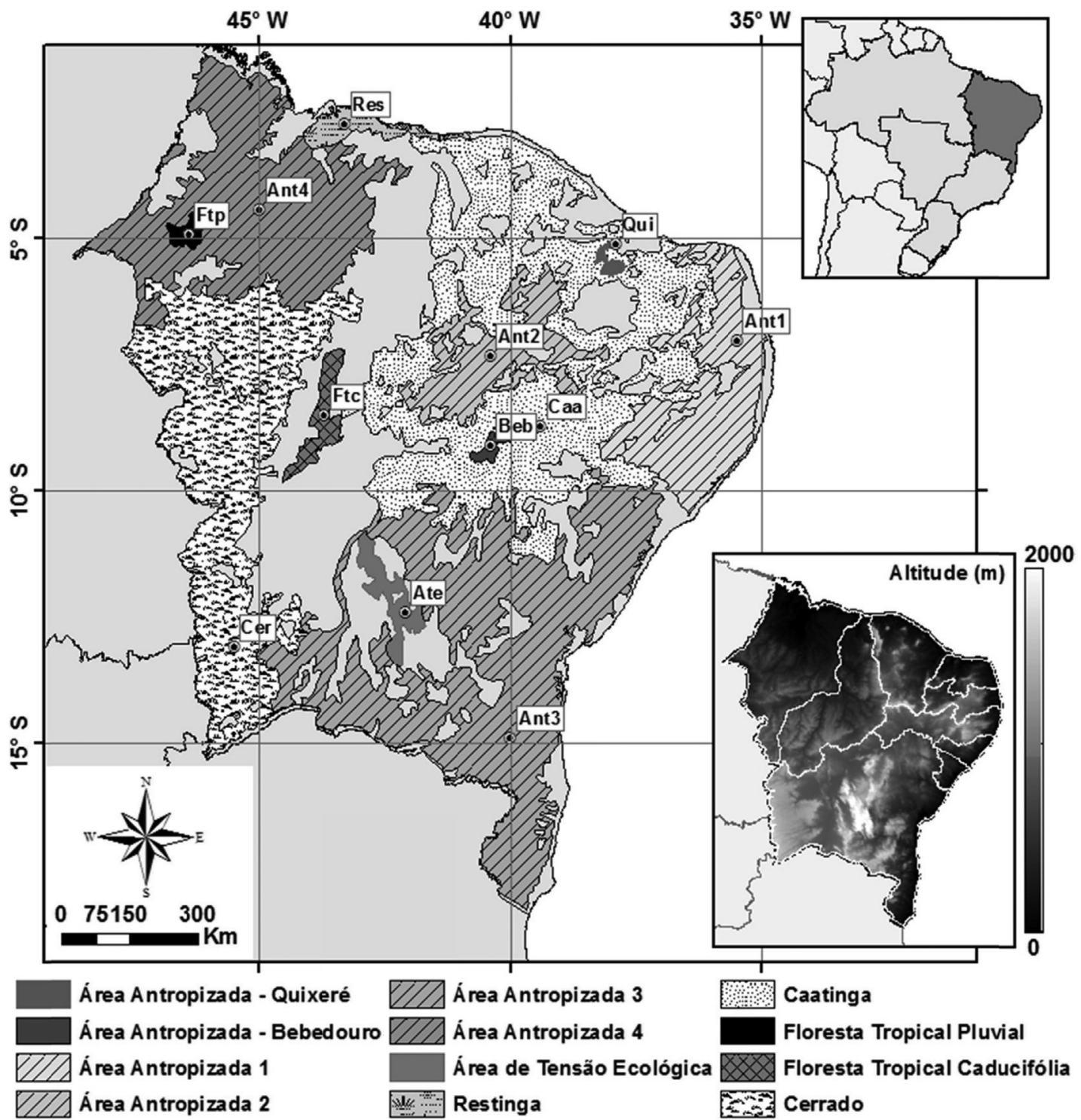

Figura 1 - Representação da Região Nordeste do Brasil apresentando sua localização, seu relevo e as respectivas áreas de estudo com seus tipos de vegetação associados. Fonte: do autor (utilizando shapefile mapa de vegetação do Brasil, IBGE - 2002).

estados de Minas Gerais e Espírito Santo e a oeste com os estados do Pará, Tocantins e Goiás. Apresenta média anual de temperatura entre $20^{\circ} \mathrm{C}$ e $28^{\circ} \mathrm{C}$. Nas áreas situadas acima de $200 \mathrm{~m}$ e no litoral oriental as temperaturas variam de $24{ }^{\circ} \mathrm{C}$ a $26{ }^{\circ} \mathrm{C}$. As médias anuais inferiores a $20{ }^{\circ} \mathrm{C}$ encontram-se nas áreas mais elevadas da Chapada Diamantina e do planalto da Borborema. Com precipitação pluviométrica média de $300 \mathrm{~mm}$ por ano em algumas regiões, as quais ocorrem durante no máximo três meses, proporcionando estiagens que duram às vezes mais de dez meses (IBGE, 2014). O NEB encontra-se com mais de $60 \%$ de seu território dentro do semiárido, segundo dados oficiais do Ministério da Integração Nacional atualizados em 2005.

Esta região possui quatro tipos de clima: (i) Clima Equatorial Úmido: presente em uma pequena parte do Estado do Maranhão e na divisa com o Estado do Pará; (ii)
Clima Litorâneo Úmido: presente do litoral do Estado da Bahia ao Rio Grande do Norte; (iii) Clima Tropical: presente nos Estados da Bahia, Ceará, Maranhão e Piauí e (iv) Clima Tropical Semiárido: presente em todo o sertão nordestino (IBGE, 2014).

As áreas de estudo utilizadas no presente trabalho foram obtidas com base no mapa de vegetação do Brasil (IBGE - 2002), disponível no site do Ministério do Meio Ambiente. Este mapa mostra as principais classes de cobertura vegetal do país, assinalando as formações remanescentes, que correspondem à vegetação que permanece preservada ou pouco alterada, e os antropismos, ou seja, as áreas afetadas pelas atividades humanas. Também traz a indicação das áreas das formações pioneiras, as áreas de tensão ecológica e os refúgios vegetacionais. 
Com base no mapa de vegetação foram selecionadas 12 áreas de interesse (AOI's) que correspondem a diferentes tipos de vegetação e de áreas antropizadas. Estas foram escolhidas de modo que englobassem a maior parte das características dos tipos de vegetação predominantes no NEB e são descritas na Tabela 1 .

Para avaliação das séries de dados do Índice de Vegetação por Diferença Normalizada (NDVI), albedo, temperatura da superfície $(T s)$ e das diversas componentes do BE fez-se a seleção de um ponto localizado em cada uma das AOI's descritas anteriormente. Com isto tornou-se possível a construção de gráficos com valores médios mensais e acumulados mensais para o período de estudo. As AOI's e os respectivos pontos estão representados na Fig. 1.

\subsection{Dados MODIS/Terra}

Os dados MODIS/Terra utilizados no trabalho foram adquiridos junto à plataforma Reverb disponibilizada pela agência EOSDIS (Earth Observing System Data and Information System) pertencente à NASA. Para os resultados foram utilizadas imagens correspondentes ao período de 1 de janeiro de 2002 a 31 de dezembro de 2011 com base nos produtos de 8 -day (produto gerado a cada 8 dias com os melhores pixels do período). Foram utilizados os Tiles h13v09, h13v10, h14v09 e h14v10 para que fosse possível abranger todo o NEB.

O produto MOD11A2 empregado possui dados globais de temperatura da superfície e emissividade combinados durante 8 dias com resolução espacial de $1 \mathrm{~km}$ a partir do produto MOD11A1, que por sua vez são gravados em grades de $1 \mathrm{~km}$ com projeção sinusoidal como médias dos valores para dias de céu claro durante o período de estudo. As bandas utilizadas são descritas na Tabela 2.

Para a reflectância da superfície foi utilizado o produto MOD09A1, que é constituído de bandas com $500 \mathrm{~m}$ de resolução espacial combinadas durante 8 dias com projeção sinusoidal. Cada pixel do produto contém a melhor obser- vação possível do período selecionado com base em alta cobertura observacional, baixo ângulo de visada, remoção de nuvens ou sombra de nuvens e aerossóis. A Tabela 3 apresenta as bandas utilizadas no estudo.

Para efeito comparativo entre as metodologias foram utilizados dados mensais GLDAS (Global Land Data Assimilation System) correspondentes ao modelo CLM (Common Land Model) para o período de 2002 a 2011, obtidos junto à plataforma Giovanni. O produto MOD16A2 também foi utilizado para fins comparativos entre a ET do modelo apresentado em Mu et al. (2011) e o modelo proposto neste estudo. Devido à falta de uma rede de estações que possibilitasse a obtenção de dados de ET de forma simples e com uma resolução espacial aceitável, tornou-se essencial a utilização destes dados para análise de médias e valores da $E T$ acumulados mensal e anual.

Os dados MOD16A2 são gerados com resolução espacial de $1 \mathrm{~km}$ para o período de 8 dias, os quais representam os valores de ET acumulados no período. O produto ainda informa os valores de $L E$ para o período. Na Tabela 4 consta a descrição do produto MOD16A2.

\subsection{Algoritmo R-SSEB}

A seguir é mostrado o procedimento para estimativa das componentes do balanço de radiação instantâneo com base em produtos Terra/MODIS por meio da metodologia desenvolvida por Araújo (2014). Para aplicações em outros satélites são utilizados procedimentos semelhantes, levando em consideração as características particulares de cada banda espectral dos respectivos satélites e dos produtos disponíveis.

O $R n$, que representa a contabilização dos fluxos radiativos à superfície, foi obtido através do cômputo dos fluxos ascendentes e descendentes das radiações de onda longa e curta atuantes no sistema solo-planta-atmosfera a partir da Eq. (1) (Bastiaanssen, 1995; Morse et al., 2001; Allen et al., 2002; Silva et al., 2005):

Tabela 1 - Descrição das áreas de interesse e dos respectivos pontos.

\begin{tabular}{lll}
\hline AOI & Sigla & Descrição \\
\hline Bebedouro & Beb & Área antropizada no interior de Pernambuco \\
Quixeré & Qui & Área antropizada do Ceará \\
Área Antropizada 1 & Ant1 & Rio Grande do Norte, Paraíba, Pernambuco e Alagoas \\
Área Antropizada 2 & Ant2 & Semiárido nordestino do Piauí, Ceará e Pernambuco \\
Área Antropizada 3 & Ant3 & Grande parte da Bahia e do Sergipe \\
Área Antropizada 4 & Ant4 & Maranhão \\
Área de Tensão Ecológica & Ate & Interior da Bahia \\
Restinga & Res & Litoral norte do Maranhão \\
Caatinga & Caa & Maior parte do semiárido nordestino \\
Floresta Tropical Pluvial & Ftp & Interior do Maranhão \\
Floresta Tropical Caducifólia & Ftc & Interior do Piauí \\
Cerrado & Cer & Oeste da Bahia e sul do Maranhão e do Piauí \\
\hline
\end{tabular}


Tabela 2 - Descrição das bandas do produto MOD11A2.

\begin{tabular}{llcc}
\hline Banda & Descrição & Unidades & Fator de escala \\
\hline 1 & $\begin{array}{l}\text { Temperatura da } \\
\text { superfície diurna }\end{array}$ & Kelvin & $0,02 * \mathrm{~B} 1$ \\
5 & $\begin{array}{l}\text { Temperatura da super- } \\
\text { fície noturna }\end{array}$ & Kelvin & $0,02 * \mathrm{~B} 2$ \\
9 & Emissividade Band 31 & - & $0,002 * \mathrm{~B} 9+0,49$ \\
10 & Emissividade Band 32 & - & $0,002 * \mathrm{~B} 10+0,49$ \\
11 & Dias de céu claro & - & - \\
12 & Noites de céu claro & - & - \\
\hline
\end{tabular}

$$
R n=R_{S \downarrow}-\alpha R_{S \downarrow}+R_{L \downarrow}-R_{L \uparrow}-\left(1-\varepsilon_{0}\right) R_{L \downarrow}
$$

$\operatorname{com} R_{S \downarrow}=$ radiação de onda curta incidente $\left(\mathrm{Wm}^{-2}\right) ; \alpha=$ albedo da superfície (adimensional); $R_{L \downarrow}=$ radiação de onda longa incidente $\left(\mathrm{Wm}^{-2}\right) ; R_{L \uparrow}=$ radiação de onda longa emitida $\left(\mathrm{Wm}^{-2}\right)$ e $\varepsilon_{0}=$ emissividade termal à superfície (adimensional). O termo $\left(1-\varepsilon_{0}\right) R_{L \downarrow}=$ representa a fração da radiação de onda longa incidente que é refletida pela superfície.

A radiação solar de onda curta incidente, como radiação direta e difusa na superfície da Terra $\left(\mathrm{Wm}^{-2}\right)$, representa a principal fonte de energia para a $E T$ e é estimada a partir da Eq. (2):

$$
R_{S \downarrow}=\frac{G_{s c} \cos \theta_{h o r} \tau_{s w}}{d^{2}}
$$

com $G_{S C}=$ constante solar $\left(1367 \mathrm{Wm}^{-2}\right) ; \theta_{\text {hor }}=$ ângulo zenital solar para superfície horizontal; $d^{2}=$ quadrado da distância relativa Terra-Sol e $\tau_{s w}=$ transmissividade atmosférica (adimensional).

A transmissividade $\tau_{s w}$ foi calculada de forma simples com auxílio do modelo de elevação digital (DEM - Digital Elevation Model) usando a Eq. (3) proposta por (Allen et al., 1998):

$$
\tau_{s w}=0,75+2 \times 10^{-5} z
$$

Tabela 4 - Descrição das bandas do produto MOD16A2.

\begin{tabular}{llcc}
\hline Banda & Descrição & Unidades & Fator de escala \\
\hline 1 & Evapotranspiração & $\mathrm{mm} / 8 \mathrm{dias}$ & 0,1 \\
2 & Fluxo de calor latente & $\mathrm{J} / \mathrm{m}^{2}$.dia & $10^{4}$ \\
3 & Evapotranspiração potencial & $\mathrm{mm} / 8 \mathrm{dias}$ & 0,1 \\
4 & Fluxo de calor latente potencial & $\mathrm{J} / \mathrm{m}^{2}$.dia & $10^{4}$ \\
\hline
\end{tabular}

$\operatorname{com} z=$ elevação do pixel (m), que pode ser representado por uma carta de DEM.

O produto MOD09A1 possui uma banda específica com os valores de $\theta_{\text {hor }}$ para cada pixel. Para efeito de simplicidade, muitos algoritmos consideram $\theta_{\text {hor }}$ constante para toda a cena, porém esta consideração somente é válida se a região de estudo possuir pouca variação de relevo e suas dimensões forem pequenas. Para regiões onde o aspecto e inclinação da superfície possuem grande variação sugere-se a aplicação da metodologia proposta por Allen et al. (2006) onde $\theta_{\text {hor }}$ deve ser substituído por $\theta_{\text {rel }}$ (ângulo zenital para superfície inclinada) tal qual determina-se utilizando a Eq. (4):

$$
\begin{aligned}
& \cos \theta_{r e l}=\sin (\delta) \sin (\varphi) \cos (s) \\
& -\sin (\delta) \cos (\varphi) \sin (s) \cos (\gamma) \\
& +\cos (\delta) \cos (\varphi) \cos (s) \cos (\omega) \\
& +\cos (\delta) \sin (\varphi) \operatorname{sen}(s) \cos (\gamma) \cos (\omega) \\
& +\cos (\delta) \sin (\gamma) \sin (s) \sin (\omega)
\end{aligned}
$$

$\operatorname{com} \delta=$ declinação da Sol (positiva no verão no hemisfério norte); $\varphi=$ latitude do pixel (positiva no hemisfério norte e negativa no hemisfério sul); $s=$ inclinação da superfície, $\operatorname{com} s=0$ ara horizontal e $s=\pi / 2$ radianos para vertical ( $s$ é sempre positivo e representa a inclinação em qualquer direção); $\gamma=$ ângulo de aspecto da superfície, $\operatorname{com} \gamma=0$ para orientação em direção ao sul, $\gamma=-\pi / 2$ para o leste, $\gamma=\pi / 2$ para o oeste e $\gamma= \pm \pi$ para o norte (valores em radianos);

Tabela 3 - Descrição das bandas do produto MOD09A1.

\begin{tabular}{llcc}
\hline Banda & Descrição & Unidades & Fator de Escala \\
\hline 1 & Reflectância $(620-670 \mathrm{~nm})$ & - & 0,001 \\
2 & Reflectância $(841-876 \mathrm{~nm})$ & - & 0,001 \\
3 & Reflectância $(459-479 \mathrm{~nm})$ & - & 0,001 \\
4 & Reflectância $(545-565 \mathrm{~nm})$ & - & 0,001 \\
5 & Reflectância $(1230-1250 \mathrm{~nm})$ & - & 0,001 \\
6 & Reflectância $(1628-1652 \mathrm{~nm})$ & - & 0,001 \\
7 & Reflectância $(2105-2155 \mathrm{~nm})$ & - & 0,001 \\
9 & Ângulo zenital solar & Graus & 0,01 \\
11 & Ângulo azimutal relativo & Graus & 0,01 \\
13 & Dia do Ano & Dia Juliano & - \\
\hline
\end{tabular}


$\omega$ = ângulo horário, com $\omega=0$ ao meio-dia, negativo pela manhã e positivo à tarde.

O parâmetro $d^{2}$ (distância relativa Terra-Sol) foi calculado em função do dia sequencial do ano $(D S A)$ usando a metodologia de Duffie e Beckman (1991) e determinada pela Eq. (5):

$$
d^{2}=\frac{1}{1+0,033 \cos (D S A \cdot 2 \pi / 365)}
$$

com $D S A=$ dia sequencial do ano e $(D S A 2 \pi / 365)$ dado em radiano. O valor do $D S A$ para cada pixel pode ser encontrado no produto MOD09A1.

O albedo foi calculado a partir da integração das reflectâncias das bandas utilizando uma função de ponderação. O produto MOD09A1 fornece os valores da reflectância à superfície os quais podem ser aplicados na Eq. (6):

$$
\alpha=\sum_{b=1}^{n}\left[\rho_{s, b} W_{b}\right]
$$

com $W_{b}=$ coeficiente de ponderação (Tabela 5), que representa a fração da radiação solar que ocorre na faixa espectral da referida banda $b$.

O NDVI é dado pela Eq. (7) como sendo uma relação entre a reflectividade das bandas 1 e 2 do Terra/MODIS, que correspondem às bandas do vermelho e do infravermelho próximo, respectivamente:

$$
N D V I=\frac{\rho_{2}-\rho_{1}}{\rho_{2}+\rho_{1}}
$$

A radiação de onda longa emitida pela superfície $\left(\mathrm{Wm}^{-2}\right.$ ) dada pela Eq. (8) foi obtida a partir da temperatura e da emissividade da superfície segundo a lei de StefanBoltzmann:

$$
R_{L \uparrow}=\varepsilon_{0} \sigma T_{S}^{4}
$$

com $\varepsilon_{0}=$ emissividade da superfície (adimensional); $\sigma=$ constante de Stefan-Boltzmann $\left(5,67 \times 10^{-8} \mathrm{~W} \mathrm{~m}^{-2} \mathrm{~K}^{-4}\right)$; $T_{S}=$ temperatura da superfície $(\mathrm{K})$. O valor de $\varepsilon_{0}$ pode ser obtido por meio da média das emissividades das bandas 31 e 32 disponíveis no produto MOD11A2.

A radiação de onda longa incidente é dada pelo fluxo termal de radiação oriundo da atmosfera $\left(\mathrm{Wm}^{-2}\right)$, sendo tradicionalmente computado usando a Eq. (9) como proposta por Stefan-Boltzmann:

$$
R_{L \downarrow}=\varepsilon_{a} \sigma T_{a}^{4}
$$

com $\varepsilon_{a}=$ emissividade da atmosfera (adimensional); $T_{a}=$ temperatura do ar próximo à superfície $(\mathrm{K})$.
O METRIC sugere que a temperatura da superfície $T_{S}$ de cada pixel da imagem pode ser utilizada no lugar de $T_{a}$, sugerindo que a radiação de onda longa incidente varia proporcionalmente à $T_{S}$. Em outras aplicações, pode-se utilizar um valor fixo de $T_{a}$ para toda a cena, considerando no caso $R_{L \downarrow}$ constante em toda a imagem, com $T_{a}$ igual a $T_{S}$ do pixel frio (Allen et al., 2007a). Neste estudo foi adotado o valor de $T_{S}$ para manter o modelo simplificado.

Uma equação empírica para $\varepsilon_{a}$ desenvolvida por Bastiaanssen (1995) pode ser aplicada para o NEB utilizando os coeficientes propostos por Araújo et al. (2012a), a partir da Eq. (10):

$$
\varepsilon_{a}=0,9565\left(-\ln \tau_{s w}\right)^{0,1362}
$$

As componentes do balanço de energia são obtidas a partir estimativa do $R n$ descrita anteriormente. Inicialmente, calculou-se o fluxo de calor no solo $(G)$ através de parametrizações. Em seguida procedeu-se a determinação dos fluxos de calor sensível $(H)$ e de calor latente $(L E)$, os quais são estimados por meio da fração evaporativa $(F E)$. A seguir é apresentada a metodologia para estimativa da evapotranspiração.

O valor de $G$ foi computado segundo a Eq. (11) desenvolvida por Bastiaanssen (2000):

$G=\left[\frac{T_{s}}{\alpha}\left(0,0038 \alpha+0,0074 \alpha^{2}\right)\left(1-0,98 N D V I^{4}\right)\right] R n$

com $T_{S}=$ temperatura da superfície $\left({ }^{\circ} \mathrm{C}\right) ; \alpha=$ albedo da superfície (adimensional); NDVI = índice de vegetação (adimensional); $R n=$ saldo de radiação $\left(\mathrm{Wm}^{-2}\right)$. Para corpos d'água, nos quais $N D V I<0$, assume-se que $G=0,3 R n$ (Bezerra et al., 2008).

Senay et al. (2007) propõem que a $F E$ seja obtida a partir de uma média de três pixels quentes e três pixels frios identificados por meio de cartas de $N D V I$ e $T s$. Os pixels frios representam áreas bem vegetadas e irrigadas, que são identificados por valores baixos de $T s$ e valores altos de NDVI. Corpos d'água também podem ser utilizados para representar os pixels frios. Já os pixels quentes são representados por áreas de pouca vegetação e solo relativamente seco, tal qual podem ser identificados por valores altos de $T s$ e valores baixos de NDVI.

Com o objetivo de operacionalizar o método, este estudo propõe uma metodologia na qual um conjunto de pixels quentes e frios é obtido automaticamente a partir de

\begin{tabular}{|c|c|c|c|c|c|c|c|}
\hline Coeficiente & Banda 1 & Banda 2 & Banda 3 & Banda 4 & Banda 5 & Banda 6 & Banda 7 \\
\hline$W_{b}$ & 0,215 & 0,215 & 0,242 & 0,129 & 0,101 & 0,062 & 0,036 \\
\hline
\end{tabular}
um conjunto de características da superfície. Tais características foram selecionadas a partir da proposta de Allen et al. (2007a) para obtenção dos pixels. Observou-se por meio de uma seleção subjetiva dos pixels que os mesmos se

Tabela 5 - Valores da constante $W_{b}$ para o sensor MODIS (Tasumi et al., 2008). 
enquadram em faixas específicas de valores, e que estão diretamente relacionados ao $N D V I, T s$ e albedo. Com isto chegou-se à Tabela 6 , mostrada a seguir, que apresenta as características da superfície para escolha dos pixels.

Com o pressuposto de que os pixels quentes apresentam valores bem pequenos de $E T$ e os pixels frios representam a $E T$ máxima ao longo da área de estudo, a média de cada conjunto de pixels pode ser utilizada para calcular as $F E$ para todos os pixels da cena a partir da Eq. (12) desenvolvida por Senay et al. (2007):

$$
F E=\frac{T_{H}-T_{S}}{T_{H}-T_{C}}
$$

com $T_{H}=$ média dos pixels quentes (hot); $T_{C}=$ média dos pixels frios (cold); $T S=$ temperatura da superfície.

Uma vez determinada a $F E$, os fluxos de calor sensível $(H)$ e latente $(L E)$ foram calculados conforme as Eqs. (13) e (14) (Roerink et al., 2000; Sobrino et al., 2007):

$$
\begin{aligned}
& H=(1-F E)(R n-G) \\
& L E=F E(R n-G)
\end{aligned}
$$

A $E T$ diária $\left(E T_{24}\right)$ foi obtida diretamente a partir da $F E$ e do $R n_{24}$ pela Eq. (15):

$$
E T_{24}=\frac{86.400 F E R n_{24}}{L}
$$

$\operatorname{com} F E=$ fração evaporativa (adimensional); $R n_{24}=$ saldo de radiação diário $\left(\mathrm{W} \cdot \mathrm{m}^{-2}\right) ; L=$ calor latente de vaporização da água $\left(2,45 \cdot 10^{6} \mathrm{~J}_{\mathrm{kg}} \mathrm{kg}^{-1}\right)$.

$\mathrm{O} R n_{24}$ foi determinado por meio do Modelo Senoidal proposto por Bisht et al. (2005) e aperfeiçoado por Araújo (2010) a partir da Eq. (16):

$$
R n_{24}=\frac{R n_{\max }}{\pi}-0,08 R n_{\max }
$$

Araújo (2010) propõe que o $R n_{24}$ seja corrigido pelo produto da Eq. (16) por um fator de correção $\left(F_{C}\right)$ que visa corrigir o problema de superestimava do modelo senoidal. $O$ valor médio encontrado para o $F_{C}$ foi de 0,75 .

$\mathrm{O} R n_{\max }$ (valor máximo do Rn observado durante o dia), em W.m ${ }^{-2}$, é dado pela Eq. (17):

$$
R n_{\text {max }}=\frac{R n}{\sin \left[\left(\frac{t_{P A S S}-t_{N A S}}{t_{P O R}-t_{N A S}}\right) \pi\right]}
$$

Tabela 6 - Características da superfície para escolha dos pixels quentes e frios.

\begin{tabular}{lcc}
\hline Caraterísticas & Pixel frio & Pixel quente \\
\hline NDVI & $>0,8$ & $<0,3$ \\
Ts & $<20,{ }^{\circ} \mathrm{C}$ & $>35,{ }^{\circ} \mathrm{C}$ \\
Albedo & $<0,2$ & $>0,3$ \\
\hline
\end{tabular}

com $t_{P A S S}=$ instante da passagem do satélite; $t_{N A S}$ e $t_{P O R}=$ instantes próximos ao nascer e pôr do Sol onde $R n=0$.

\section{Resultados e Discussão}

Quando comparados os dados R-SSEB aos dados CLM observou-se que os menores erros estavam associados aos alvos Beb e Caa, que apresentaram valores abaixo de 8\%. O alvo Qui apresentou o maior EMP dentre todos os alvos, com valor superior a $48 \%$. Os demais alvos apresentaram EMP variando de $20 \%$ a $30 \%$, em média. Os EMA e EMP correspondentes foram de $181 \mathrm{~mm} /$ ano e $23,6 \%$, respectivamente. Ao compararem-se os dados do R-SSEB aos do produto MOD16 evidenciou-se que os mesmos tendem a superestimar os valores de ET obtidos neste estudo. Essas diferenças entre os modelos podem estar associadas ao fato de que o método empregado por Mu et al. (2011) é muito dependente de dados de reanálise para estimativa dos elementos do tempo e da resistência aerodinâmica. Além disso, a resistência da cultura é obtida com base em dados médios para diversos biomas em todo o globo, estes que podem comprometer a estimativa desse parâmetro. As menores diferenças foram observadas no alvo Beb, com valor médio de $9 \%$. As maiores diferenças são observadas nos alvos da região do meio-norte e nos alvos Qui e Ftc. Os EMA e EMP entre os dados R-SSEB e MOD16 foram de $288 \mathrm{~mm} /$ ano e $38,6 \%$, respectivamente.

O grau de correlação entre os modelos foi considerado significativo, apresentando um valor de 0,493 entre o R-SSEB e o CLM e valores acima de 0,70 entre o R-SSEB e MOD16 e entre o MOD16 e CLM, respectivamente. Os coeficientes de determinação (R2) variaram de 0,243 para os modelos R-SSEB e CLM a valores acima de 0,50 entre os outros pares avaliados. Mesmo se observando um grau de correlação menor entre os modelos R-SSEB e CLM, constatou-se pelo Teste F que não houve diferença significativa entre os três conjuntos de dados estudados ao nível de 5\% de significância. A Fig. 2 apresenta a matriz de dispersão entre os modelos estudados.

Autovino et al. (2016) compararam dados MOD16 com ET medida em experimento de correlações turbulentas em um distrito de irrigação localizado na Itália e obtiveram valores de $0,83 \mathrm{~mm} /$ dia e 0,23 para raiz do erro médio quadrático (RMSE) e R2, respectivamente. Hu et al. (2015) avaliaram o MOD16 sobre a Europa para o ano de 2011 e obtiveram valores de R2 variando de 0,29 em áreas de mata aberta na Espanha a 0,98 em áreas de floresta boreal. $\mathrm{O}$ RMSE variou de $0,33 \mathrm{~mm} /$ dia a $1,57 \mathrm{~mm} /$ dia. Velpuri et al. (2013) valores de R2 e RMSE iguais a 0,56 e $29 \mathrm{~mm} / \mathrm{mês}$ quando compararam o MOD16 a dados das torres de correlações turbulentas FLUXNET sobre os Estados Unidos.

Avaliando os gráficos apresentados na Fig. 2 observa-se que o MOD16 tende a deslocar seu eixo da dispersão para valores acima de $1000 \mathrm{~mm} / \mathrm{ano}$, o que indica que o mesmo superestima os demais modelos. Quando comparamos o R-SSEB e o CLM observa-se uma maior concen- 


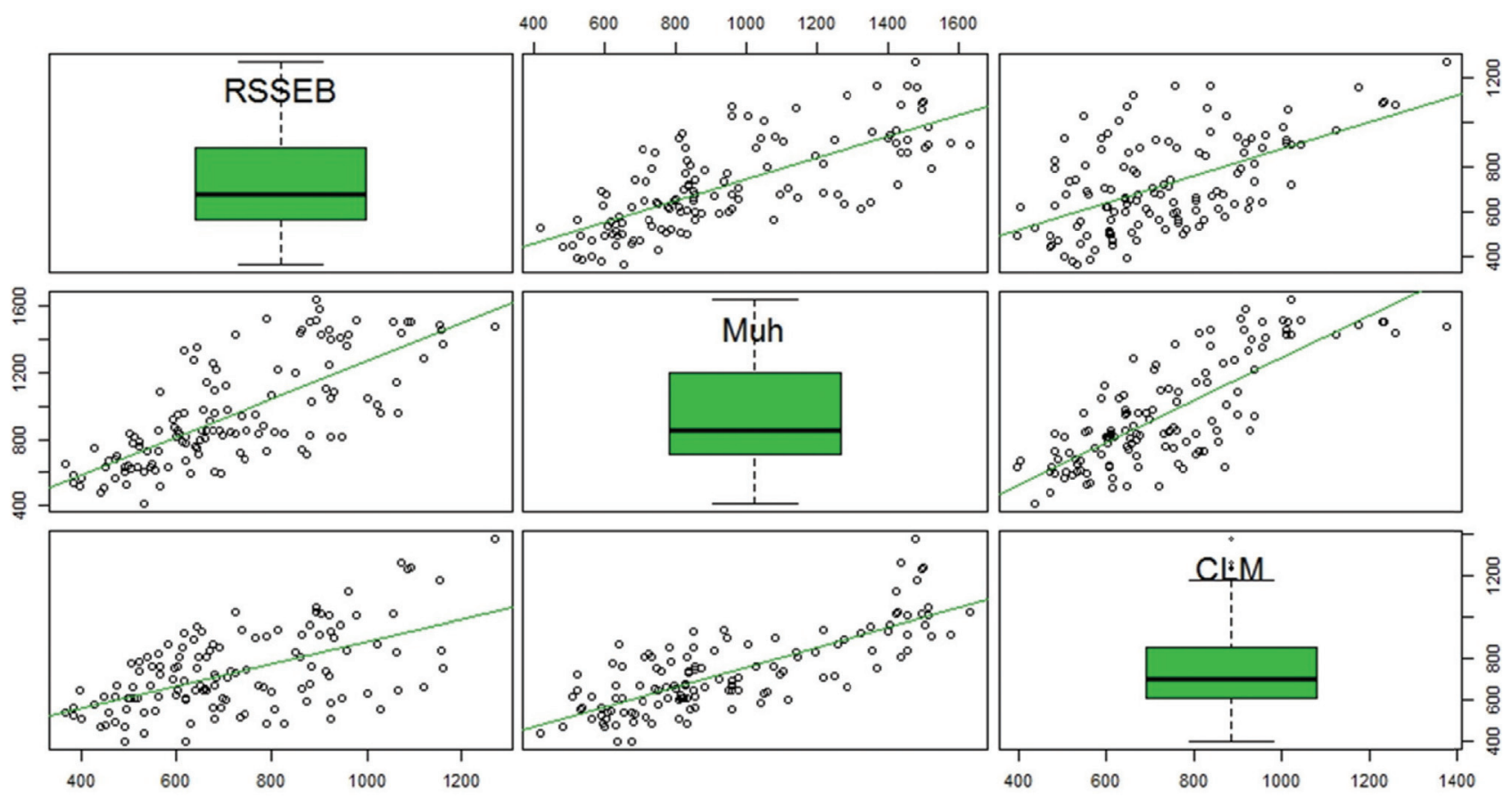

Figura 2 - Matriz de dispersão entre os dados R-SSEB, MOD16 e CLM.

tração dos dados na faixa de $E T$ inferior a $1000 \mathrm{~mm} / \mathrm{ano}$, o que indica uma maior proximidade entre os dois modelos. Analisando o conjunto de dados conclui-se, em geral, que os menores valores de ET se apresentam agrupados, enquanto os maiores valores mostram-se mais dispersos em todos os casos estudados.

Os perfis da $E T$ acumulada anual são apresentados na Fig. 3. O modelo R-SSEB apresentou a menor variação de sua mediana ao longo dos anos, seguido do CLM e do MOD16. No período de 2003 a 2006 o CLM mostra menor variabilidade em seus dados anuais, o que não foi observado nos outros modelos. O MOD16 demonstra maior variabilidade ao longo de todo o período estudado, com destaque para os anos de 2002 e 2003. O R-SSEB e o CLM apresentaram, em média, aproximadamente $75 \%$ dos seus dados abaixo da faixa dos $1000 \mathrm{~mm} / \mathrm{ano}$ ao longo do tempo, enquanto o MOD16 chegou a apresentar aproximadamente $50 \%$ dos dados acima dos $1000 \mathrm{~mm} /$ ano no período de 2009 a 2011 .

A Fig. 4 destaca os mapas da variabilidade espacial da ET (mm/ano) no período de 2002 a 2011 para o modelo proposto neste estudo (R-SSEB) e os modelos MOD16 e CLM. De acordo com os mapas nota-se que o MOD16 tende a superestimar os valores de $E T$ no Maranhão, norte do Piauí e do Ceará e no litoral nordestino. Na região do semiárido os valores de ambos os modelos se apresentam similares. O modelo CLM apresenta uma distribuição espacial similar ao MOD16 para valores de ET abaixo de $700 \mathrm{~mm} /$ ano. Já na região litorânea e no meio-norte observa-se uma proximidade entre os modelos R-SSEB e CLM.
Para análise das quatro componentes do $B E$ foram selecionados os anos com menor número de falhas nos dados para cada alvo da região de estudo. A Fig. 5 apresenta os dados de $R n, G, H$ e $L E$, em Wm${ }^{-2}$, para cada alvo a partir de dados obtidos a cada 8 dias por meio do MODIS/Terra.

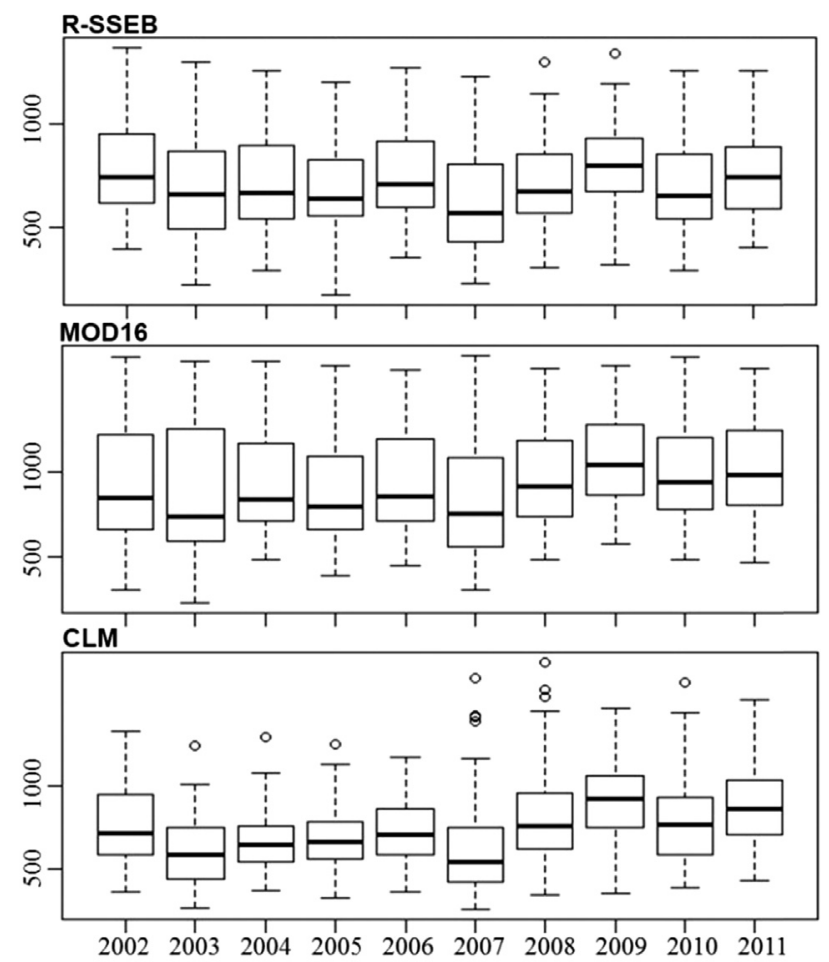

Figura 3 - Variação da ET acumulada anual (mm/ano) de 2002 a 2011 para os modelos R-SSEB, MOD16 e CLM. 

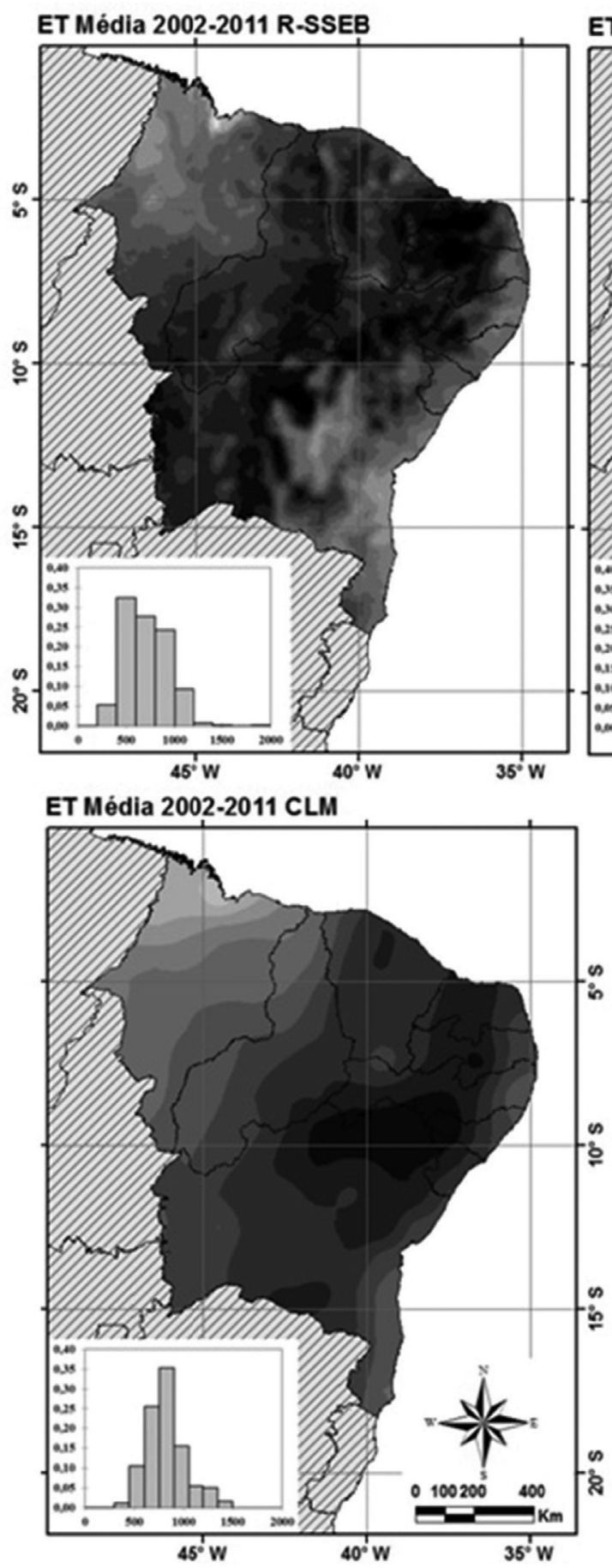

ET Média 2002-2011 MOD16
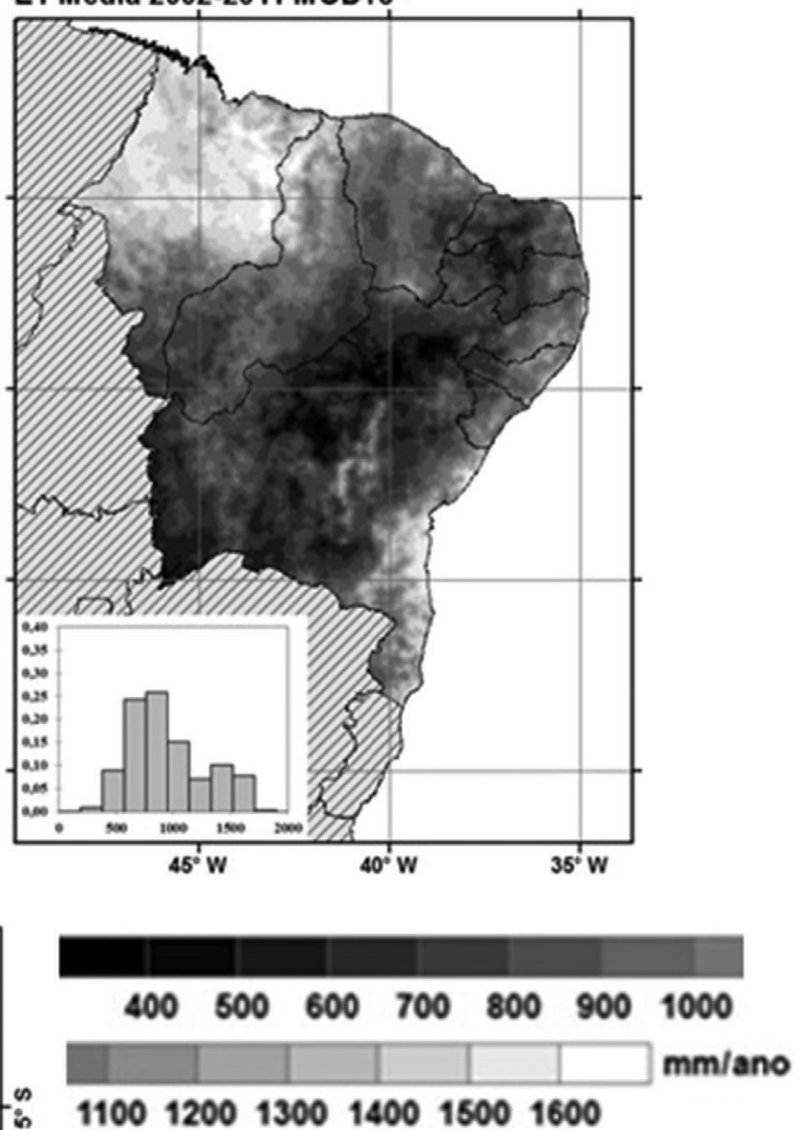

Figura 4 - ET média 2002-2011 (mm/ano) dos modelos R-SSEB, MOD16 e CLM.

Os valores de $G$ situaram-se numa faixa abaixo dos $100 \mathrm{Wm}^{-2}$ para a maioria dos alvos estudados. Contudo, os alvos Caa, Ant1 e Ant2 apresentaram variabilidade maior, chegando a atingir valores próximos a $120 \mathrm{Wm}^{-2}$. O Rn apresentou a menor variação de sua mediana ao longo dos alvos, com valores da ordem de $600 \mathrm{Wm}^{-2}$. A maior variabilidade foi observada nos alvos Cer e Ant3, onde a diferença entre os quartis foi consideravelmente maior que os demais. Os maiores picos de $R n$ foram observados nas áreas Ftp, Ftc e Cer, chegando a atingir valores próximos a $800 \mathrm{Wm}^{-2}$. A área Ant2, situada no interior do semiárido, apresentou a menor variabilidade de $R n$ ao longo do período estudado.

A relação inversa de $H$ e $L E$ permite observar a influência direta da quantidade de água disponível para $E T$ em cada alvo da área de estudo. Nos alvos Ant3 e Ftp este 
Fluxo de Calor Sensível (H)

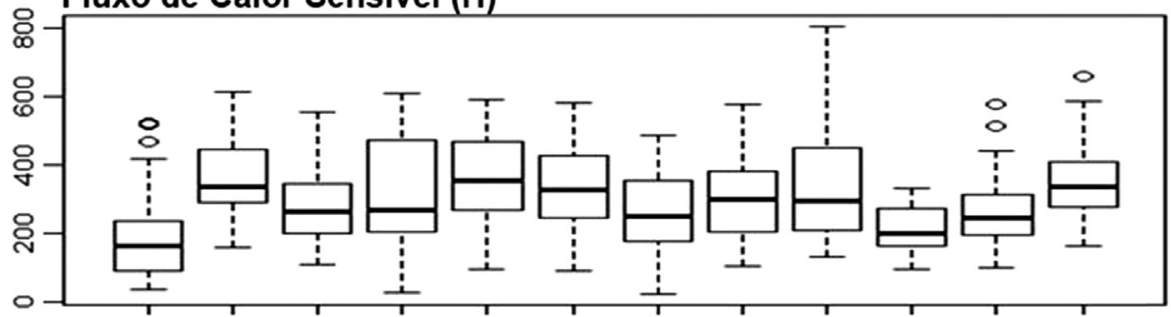

Fluxo de Calor Latente (LE)

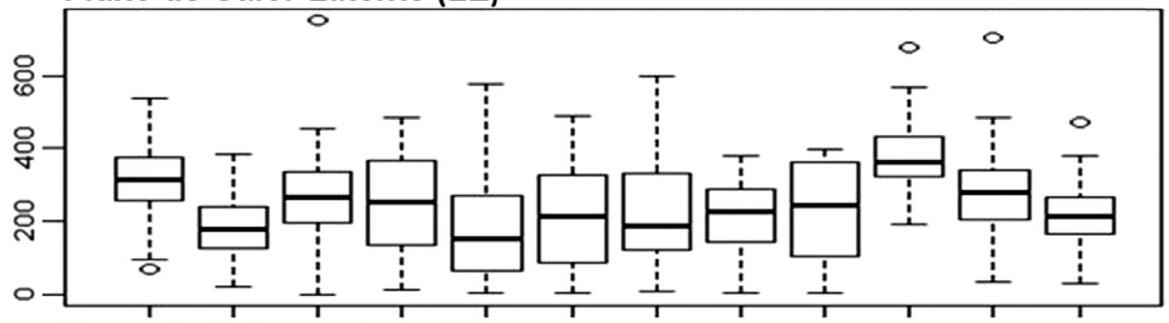

Fluxo de Calor no Solo (G)

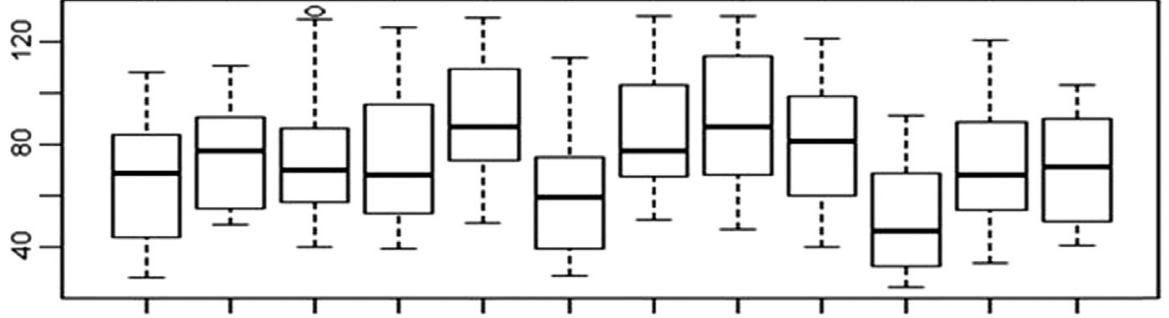

Saldo de Radiação (Rn)

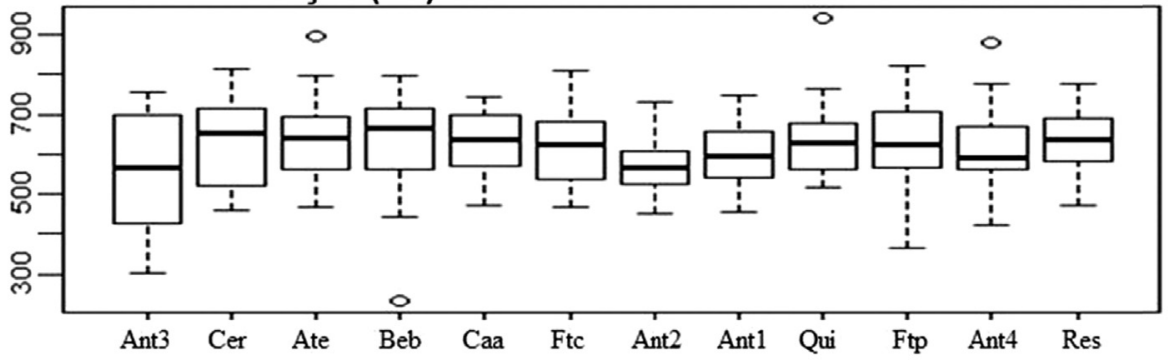

Figura 5 - Variação dos fluxos $H, L E, G$ e $R n\left(\mathrm{Wm}^{-2}\right)$ para cada alvo da área de estudo.

aspecto é evidenciando ao observar-se que o valor de $L E$ geralmente é maior que o de $H$. Já em alvos onde o período seco influencia diretamente a quantidade de água disponível, observa-se que o valor de $H$ tende apresentar a mesma variabilidade do $L E$ em algumas épocas do ano, como pode-se notar na maioria dos alvos. Para os alvos Caa e Cer, onde a vegetação é mais esparsa, pode-se verificar que o valor de $H$ supera o $L E$ na maior parte do ano. Silva (2009) encontrou uma predominância de valores de $G$ em torno de $100 \mathrm{Wm}^{-2} \mathrm{em}$ áreas de caatinga e valores na faixa de 50 a $75 \mathrm{Wm}^{-2}$ em áreas irrigadas. Obteve também valores de $L E$ variando de $300 \mathrm{a} 600 \mathrm{Wm}^{-2}$ em áreas irrigadas e inferiores a $300 \mathrm{Wm}^{-2}$ na caatinga. Santos (2009) encontrou valores médios de $L E$ superiores a $200 \mathrm{Wm}^{-2}$ sobre plantação de bananas em Quixeré - CE.

A ET é função direta da $F E$ e do $R n_{24 h}$ como pode ser observado na Equação 15. A metodologia original empre- gada por Senay et al. (2007) indica a escolha de 3 pixels quentes e 3 pixels frios para o cálculo de uma média, esta que é empregada na Equação 12 para obtenção da $F E$ ponto a ponto. Neste estudo foi proposta uma metodologia operacional que permitisse a escolha automática destes pixels âncoras. Observou-se que, em média, foram selecionados menos que $0,5 \%$ da quantidade de pixels totais das cenas MODIS/Terra empregadas no estudo. As cenas apresentaram um total de 12.793 pontos com grade de $10 \mathrm{~km}$.

A Tabela 7 apresenta os valores acumulados da $E T$ para o período de um ano para cada alvo da região de estudo. Os maiores valores foram encontrados nos alvos Ant 3 e Ftp, com valores de ET superiores a 1.000 mm/ano em praticamente todo o período estudado. Os menores valores foram observados no alvo Ftc, que possui por característica principal a queda de folhas de sua vegetação para evitar perdas de aguas excessivas no período seco. 
Tabela 7 - Valores anuais acumulados de $E T$ (mm/ano) para a região Nordeste.

\begin{tabular}{lcccccccccc}
\hline Ponto & 2002 & 2003 & 2004 & 2005 & 2006 & 2007 & 2008 & 2009 & 2010 & 2011 \\
\hline Ant3 & 1100 & 1008 & 1053 & 916 & 1017 & 1043 & 977 & 1046 & 903 & 1117 \\
Cer & 524 & 616 & 644 & 723 & 747 & 454 & 547 & 628 & 628 & 598 \\
Ate & 1028 & 580 & 782 & 902 & 973 & 724 & 747 & 848 & 800 & 816 \\
Beb & 800 & 603 & 466 & 522 & 541 & 334 & 389 & 657 & 420 & 650 \\
Caa & 658 & 334 & 464 & 504 & 460 & 473 & 484 & 597 & 516 & 467 \\
Ftc & 496 & 494 & 473 & 589 & 611 & 415 & 625 & 880 & 439 & 593 \\
Ant2 & 654 & 461 & 544 & 384 & 474 & 410 & 512 & 551 & 545 & 625 \\
Ant1 & 826 & 918 & 913 & 1003 & 772 & 979 & 955 & 1108 & 1032 & 958 \\
Qui & 636 & 330 & 452 & 337 & 419 & 317 & 357 & 537 & 313 & 546 \\
Ftp & 1113 & 1138 & 1215 & 1060 & 1125 & 1087 & 1110 & 1120 & 1018 & 1073 \\
Ant4 & 826 & 903 & 783 & 685 & 870 & 781 & 738 & 844 & 772 & 766 \\
Res & 818 & 994 & 866 & 847 & 902 & 831 & 884 & 1007 & 985 & 871 \\
\hline
\end{tabular}

Os alvos do semiárido Beb, Caa, Ant2 e Qui apresentaram comportamento similar entre si, com valores de $E T$ oscilando, em média, de 300 a $650 \mathrm{~mm} /$ ano. A restinga apresentou um comportamento de $E T$ variando de 818 a $1007 \mathrm{~mm} / \mathrm{ano}$, enquanto no cerrado variou de $454 \mathrm{a}$ $747 \mathrm{~mm} / \mathrm{ano}$.

Na Fig. 6 são apresentados os valores médios da $E T$ acumulada mensalmente para o período de 2002 a 2011 para os 12 alvos espalhados pelo NEB. Observa-se uma forte relação entre os valores de $E T$ e o período chuvoso de cada região. Os alvos localizados no semiárido e no cerrado possuem maior influência do período seco, tendo seus valores mínimos situados abaixo dos $20 \mathrm{~mm} / \mathrm{mês}$. $\mathrm{O}$ alvo Ftc apresenta valores extremamente baixos de $E T$ no período seco, devido ao fato de que suas árvores perdem as folhas nesse período para evitar a perda excessiva de água. Os alvos Ant3, Ant1, Ftp e Res apresentam as maiores medias de $E T$ devido à maior presença de água disponível em suas respectivas áreas. Os alvos da Caa e Ant tem um comportamento similar ao alvo Cer, porém observa-se que este último possui um período seco menor.

Em março e abril foram observados valores de $E T$ superiores a $120 \mathrm{~mm} / \mathrm{mês}$ para os alvos Ate, Ftp e Res. Os valores de $E T$ alvo Ant 3 situaram-se acima dos $60 \mathrm{~mm} / \mathrm{mês}$ em todo o ano. O trimestre jul/ago/set apresentou os menores valores de $E T$ para o cerrado e as regiões do semiárido, com valores abaixo dos $20 \mathrm{~mm} / \mathrm{mês}$ em vários alvos. As amplitudes máximas observadas foram de ordem de $80 \mathrm{~mm} / \mathrm{mês}$ para alguns alvos, tais como Ftc e Res. Os alvos
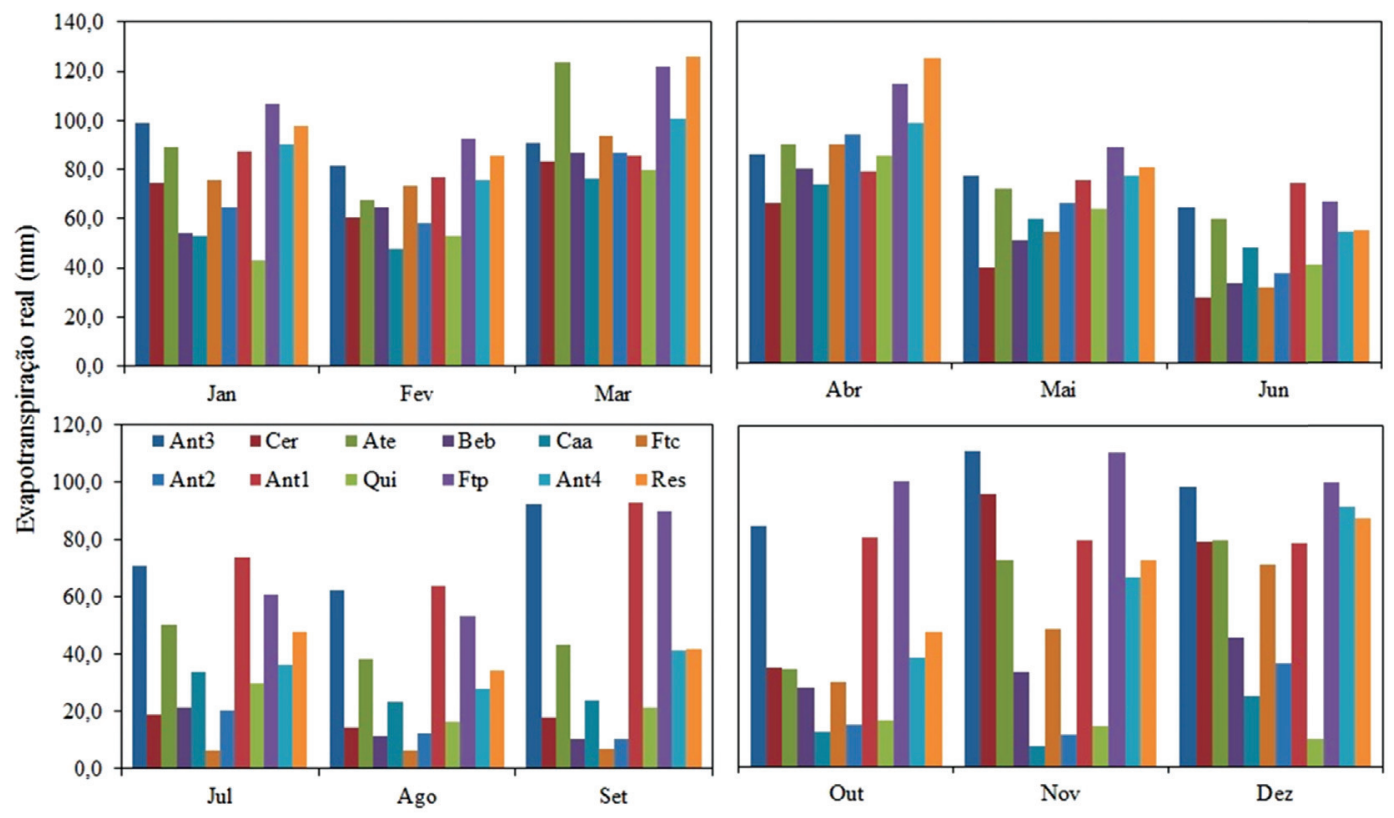

Figura 6 - ET média mensal (mm/mês) (2002-2011) para alvos da região Nordeste. 
Ant3 e Ftp apresentaram as menores variações de $E T$ ao longo do ano.

\section{Conclusões}

Com o exposto conclui-se que o modelo proposto neste estudo mostrou-se bastante promissor, permitindo o processamento de uma grande quantidade de dados de forma simplificada e operacional, sem comprometer a precisão das informações obtidas.

Os dados de ET anuais foram comparados aos modelos GLDAS e Mu et al. (2011) como forma de validação. Foram observados EMA e EMP de $181 \mathrm{~mm} / \mathrm{ano}$ e 23,6\% entre os modelos R-SSEB e GLDAS, respectivamente. Quando comparados os modelos R-SSEB e Mu et al. (2011) foram observados EMA e EMP de $288 \mathrm{~mm} / \mathrm{ano}$ e $38,6 \%$, respectivamente. Por meio do Teste F constatou-se que não houve diferença significativa entre os modelos ao nível de 5\% de significância. Os valores de R2 encontrados entre os modelos mostram-se condizentes com valores encontrados na literatura para outras partes do mundo.

A seleção automática dos pixels âncoras por meio de parâmetros pré-definidos de $N D V I$, albedo e $T S$ se mostrou bastante consistente, permitindo uma seleção confiável dos pixels quentes e frios. A quantidade de pixels selecionada para o cômputo da fração evaporativa correspondeu a menos de $0,5 \%$ do número total de pixels da região como um todo. Os dados médios anuais de $E T$ variaram de 482 a $1105 \mathrm{~mm} . \mathrm{ano}^{-1} \mathrm{em}$ áreas de caatinga e floresta tropical pluvial, respectivamente. Quando comparados a dados de ET gerados segundo Mu et al. (2011) observou-se que houve uma superestimativa por parte destes últimos, com variação de 596 a 1532 mm.dia ${ }^{-1}$ para as mesmas áreas citadas. Observou-se que essa superestimativa variou de $9 \% \mathrm{em}$ um alvo do semiárido até valores próximos a $80 \% \mathrm{em}$ alvos de floresta tropical.

Os resultados evidenciam que a metodologia proposta constitui uma poderosa ferramenta para estimativa das componentes do $B E$ e da $E T$ não só em grande escala, mas também em escala local. O modelo proposto neste estudo se apresenta como uma excelente alternativa às metodologias existentes, por ser facilmente adaptável a calibrações locais e por permitir a obtenção de produtos derivados do $B E$ e da $E T$ com facilidade e boa precisão. Contudo, sugere-se que sejam realizadas validações em experimentos de campo em diversas áreas da região Nordeste para verificação da acurácia do modelo proposto neste estudo.

\section{Agradecimentos}

Os autores agradecem ao Conselho Nacional de Desenvolvimento Científico e Tecnológico (CNPq) (Projetos: 471497/2012-6, 446172/2015-4 e 301348/2015-4), pelo apoio financeiro a esta pesquisa e pela concessão das bolsas de Produtividade em pesquisa aos autores, à Coordenação de Aperfeiçoamento de Pessoal de Nível Superior (CAPES) (Projeto Pró-Alertas - 88887.091737/2014-01), pela concessão das bolsas de estudo, e a Campina Geotech Consultoria em Meteorologia Ltda.; pela parceria na elaboração do artigo.

\section{Referências}

AGAM, N. ; KUSTAS, W.P. ; ANDERSON, M.C.; LI, F.; NEALE, C.M.U. A vegetation index based technique for spatial sharpening of thermal imagery. Remote Sensing of Environment, v. 107, p. 545-558, 2007.

ALLEN, R.G.; TASUMI, M.; TREZZA, R. Satellite-based energy balance for mapping evapotranspiration with internalized calibration (METRIC) - Model. Journal of Irrigation and Drainage Engineering, v. 133, p. 380-394, 2007 a.

ALLEN, R.G.; TASUMI, M.; TREZZA, R. Satellite-based energy balance for mapping evapotranspiration with internalized calibration (METRIC) - Applications. Journal of Irrigation and Drainage Engineering, v. 133, p. 395-406, 2007b.

ALLEN, R.G.; TREZZA, R.; TASUMI M. Surface energy balance algorithms for land. Advance training and user's manual, version 1.0, pp. 98, 2002.

ALLEN, R.G.; TREZZA, R.; TASUMI, M. Analytical integrated functions for daily solar radiation on slopes. Agricultural and Forest Meteorology, v. 139, n. 1, p. 55-73, 2006.

ALLEN, R.G.; PEREIRA, L.S.; RAES, D.; SMITH, M. Crop evapotranspiration: Guidelines for computing crop water requeriments. United Nations FAO, Irrigation and Drainage, N.Y.; Paper No. 56, 1998.

ALLEN, R.; TASUMI, M.; TREZZA, R.; BASTIAANSSEN, W.; MORSE, T. et al. Metric: High Resolution Satellite Quantification of Evapotranspiration. Presentation Part Two - Energy Balance. Workshop. University of Idaho. Kimberley-Id, 2005.

ARAÚJO, A.L. Calibração do balanço de radiação diário através de dados de superfície e de sensores orbitais. $114 \mathrm{f}$. Dissertação (Mestrado em Meteorologia). Universidade Federal de Campina Grande, Campina Grande, Paraíba, 2010.

ARAÚJO, A.L.; SILVA, B.B.; BRAGA, C.C. Simplified modeling of downwelling long-wave radiation over Brazilian semi-arid under irrigation conditions. Revista Brasileira de Geofísica, v. 30, p. 137-145, 2012a.

ARAÚJO, A.L.; SANTOS, C.A.C.; SILVA, B.B.; BEZERRA, B.G.; BORGES, V.P. Refinamento de imagens termais do Landsat 5-TM com base em classes de NDVI. Revista Brasileira de Meteorologia, v. 27, p. 470-476, 2012 b.

ARAÚJO, A.L. Operacionalização do balanço de energia e evapotranspiração em escala regional com dados de sensores orbitais. 101f. Tese (Doutorado em Meteorologia). Universidade Federal de Campina Grande, Campina Grande, Paraíba, 2014.

AUTOVINO, D.; MINACAPILLI, M.; PROVENZANO, G. Modelling bulk surface resistance by MODIS data and assessment of MOD16A2 evapotranspiration product in an irrigation district of Southern Italy. Agricultural Water Management, v. 167, p. 86-94, 2016.

BASTIAANSSEN, W.G.M. Regionalization of surface flux densities and moisture indicators in composite terrain: A remote sensing approach under clear skies in Mediterra- 
nean climates. Ph.D. Dissertation, CIP Data Koninklijke Bibliotheek, Den Haag, The Netherlands. 1995.

BASTIAANSSEN, W.G.M. SEBAL-based sensible and latent heat fluxes in the irrigated Gediz Basin, Turkey. Journal of Hydrology, v. 229, p. 87-100, 2000.

BASTIAANSSEN, W.G.M.; MENENTI, M.; FEDDES, R.A.; HOLTSLAG, A.A.M. A remote sensing surface energy balance algorithm for land (SEBAL). 1: Formulation. Journal Hydrology, v. 212-213, p. 198-212, 1998a.

BASTIAANSSEN, W.G.M.; PELGRUM, H.; WANG, J.; MA, Y.; MORENO, J.F. et al. A remote sensing surface energy balance algorithm for land (SEBAL). 1: Validation. Journal Hydrology, v. 212-213, p. 213-229, 1998b.

BASTIAANSSEN, W.G.M.; NOORDMAN, E.J.M.; PELGRUN, H.; DAVIDS, G.; THORESON, B.P. et al. SEBAL Model with Remotely Sensed Data to Improve Water-Resources Management under Actual Field Conditions. Journal of Irrigation and Drainage Engineering, ASCE, p. 85-93, 2005.

BEZERRA, B.G.; SILVA, B.B.; FERREIRA, N. J. Estimativa da evapotranspiração diária utilizando-se imagens digitais TM - Landsat 5. Revista Brasileira de Meteorologia, v. 23, p. 305-317, 2008.

BISHT, G.; VENTURINI, V. ; ISLAM, S.; JIANG, L. Estimation of the net radiation using MODIS (Moderate Resolution Imaging Spectroradiometer) data for clear-sky days. Remote Sensing of Environment, v. 97, p. 52-67, 2005.

DI PACE, F.T.; SILVA, B.B.; SILVA, V. P. R.; SILVA, S.T.A. Mapeamento do saldo de radiação com imagens Landsat 5 e modelo de elevação digital. Revista Brasileira de Engenharia Agrícola e Ambiental, v. 12, p. 385-392, 2008.

DUFFIE, J.A.; BECKMAN, W.A. Solar engineering of thermal process, $2^{\text {nd }}$ Ed.; Wiley, N. Y. 1991.

GAO, Z.Q.; LIU, C.S.; GAO, W.; CHANG, N. B. A coupled remote sensing and the Surface Energy Balance with Topography Algorithm (SEBTA) to estimate actual evapotranspiration over heterogeneous terrain. Hydrology and Earth System Sciences, v. 15, p. 119-139, 2011.

HU, G.; JIA, L.; MENENTI, M. Comparison of MOD16 and LSA-SAF MSG evapotranspiration products over Europe for 2011. Remote Sensing of Environment, v. 156, p. 510526, 2015.

IBGE. Instituto Brasileiro de Geografia e Estatística. Disponível em http:/www.ibge.gov. br/. Acesso em 10 de fevereiro de 2014.

KITE, G.W.; DROOGERS, P. Comparison evapotranspiration estimates from satellites, hydrological models and field data, Journal of Hydrology, v. 229, p3-18, 2000.

MENDONÇA, J.C; SOUSA, E.F; BOUHID, R.G; SILVA, B.B; FERREIRA, N. J. Estimativa do fluxo do calor sensível utilizando o algoritmo sebal e imagens modis para a região norte fluminense, RJ. Revista Brasileira de Meteorologia, v. 27, p. 85-94, 2012.

MORSE, A.; ALLEN, R.G.; TASUMI, M.; KRAMBER, W.J.; TREZZA, R. et al. Application of the SEBAL Methodology for Estimating Evapotranspiration and Consumptive Use of Water Through Remote Sensing. Final Report, 220 p, 2001.

MU, Q.; HEINSCH, F.A.; ZHAO, M.; RUNNING, S.W. Development of a global evapotranspiration algorithm based on
MODIS and global meteorology data. Remote Sensing of Environment, v. 111, p. 519-536, 2007.

MU, Q.; ZHAO, M.; RUNNING, S.W. Improvements to a MODIS global terrestrial evapotranspiration algorithm. Remote Sensing of Environment, v. 115, p. 1781-1800, 2011.

NORMAN, J.M.; KUSTAS, W.P. ; HUMES, K.S. Source approach for estimating soil and vegetation energy fluxes in observations of directional radiometric surface temperature. Agricultural and Forest Meteorology. V. 77, p. 263-293, 1995.

ROERINK, G.J.; SU, Z.; MENENTI, M. A Simple Remote Sensing Algorithm to Estimates the Surface Energy Balance. Physics and Chemistry of the Earth. n. 25, p. 147-157, 2000.

SANTOS, C.A.C. Estimativa da evapotranspiração real diária através de análises micrometeorológicas e de sensoriamento remoto. 134f. Tese (Doutorado em Meteorologia). Universidade Federal de Campina Grande, Campina Grande, Paraíba, 2009.

SANTOS, C.A.C.; SILVA, B.B. Estimativa da evapotranspiração da bananeira em região semi-árida através do algoritmo S-SEBI. Revista Brasileira de Agrometeorologia, v. 16, n. 1, p. 9-20, 2008.

SANTOS, C.A.C.; SILVA, B.B.; RAMANA RAO, T.V. Estimativa da ET diária utilizando os algortimos SEBAL e S-SEBI. Ciência e Natura, v. 29, p. 71-74, 2007.

SCHUURMANS, J.M.; TROCH, P. A.; VELDHUIZEN, A.A.; BASTIAANSEEN, W.G.M.; BIERKENS, M.F.P. Assimilation of remotely sensed latent heat flux in a distributed hydrological model. Advances in Water Resources, v. 26, p. 151-159, 2003.

SENAY, G.B.; BUDDE, M.; VERDIN, J.P. ; MELESSE, A. M. A coupled remote sensing and simplified surface energy balance approach to estimate actual evapotranspiration from irrigated fields. Sensors, v. 7, p. 979-1000, 2007.

SENAY, G.B.; BOHMS, S.; SINGH, R.K.; GOWDA, P.H.; VELPURI, N. M. et al. Operational evapotranspiration mapping using remote sensing and weather datasets: a new parameterization for the SSEB approach. Journal of the American Water Resources Association, v. 49, p. 577591, 2013.

SILVA, B.B.; BEZERRA, M.V. C. Determinação dos fluxos de calor sensível e latente na superfície utilizando imagens TM - Landsat 5. Revista Brasileira de Agrometeorologia, v. 14, p. 174-186, 2006.

SILVA, B.B.; BRAGA, A.C.; BRAGA, C.C.; OLIVEIRA, L.M.M.; GALVÍNCIO, J.D. et al. Evapotranspiração e estimativa da água consumida em perímetro irrigado do semiárido brasileiro por sensoriamento remoto. Pesquisa Agropecuária Brasileira, v. 47, p. 1218-1226, 2012.

SILVA, B.B.; LOPES, G.M.; AZEVEDO, P. V. Balanço de radiação em áreas irrigadas utilizando imagens Landsat 5 - TM. Revista Brasileira de Meteorologia, v. 20, p. 243-252, 2005.

SILVA, B.B.; MENDONÇA, R.R.O.; SILVA, S.T.A.; FERREIRA, R.C. Mapeamento do albedo de áreas heterogêneas do estado do Ceará com imagens TM - Landsat 5. Revista de Geografia, v. 25, p. 33-52, 2008.

SILVA, S.T.A. Mapeamento da evapotranspiração na bacia hidrográfica do baixo Jaguaribe usando técnicas de sensoriamento remoto. 136f. Tese (Doutorado em Meteoro- 
logia). Universidade Federal de Campina Grande, Campina Grande, Paraíba, 2009.

SOBRINO, J.A.; GOMEZ, M.; JIMENEZ-MUNOZ, J.C.; OLIO$\mathrm{SO}, \mathrm{A}$. Application of a simple algorithm to estimate daily evapotranspiration from NOAA-AVHRR images for the Iberian Peninsula. Remote Sensing of Environment, v. 110, p. 139-148, 2007.

SU, Z. The Surface Energy Balance System (SEBS) for estimation of turbulent heat fluxes, Hydrology and Earth System Sciences, v. 6, p. 85-100, 2002.

TASUMI, M. Progress in operational estimation of regional evapotranspiration using satellite imagery. Ph.D. dissertation, Univ. of Idaho, Moscow, Id. 2003.

TASUMI, M.; ALLEN, R.G.; TREZZA, R. At-surface reflectance and albedo from satellite for operational calculation of land surface energy balance. Journal of Hydrologic Engineering, v. 13, p. 51-63, 2008.

TEIXEIRA, A.H.C. Modelling evapotranspiration by remote sensing parameters and agro-meteorological stations. In:
NEALE, C. M. U.; COSH, M. H. (Org.). Remote Sensing and Hydrology, v. 352, p. 154-157, 2012.

TREZZA, R. Evapotranspiration using a satellite-based Surface energy balance with Standardized ground control. 247f. Thesis (Doctor of Philosophy in Biological and Agricultural Engineering). Utah State University. Logan, Utah, 2002.

VELPURI, N. M.; SENAY, G.B.; SINGH, R.K.; BOHMS, S.; VERDIN, J.P. A comprehensive evaluation of two MODIS evapotranspiration products over the conterminous United States: Using point and gridded FLUXNET and water balance ET. Remote Sensing of Environment, v. 139, p. 35-49, 2013.

\section{Endereços de Internet}

http://mapas.mma.gov. br/i3geo/datadownload.htm reverb.echo.nasa.gov

giovanni.gsfc.nasa.gov/giovanni

This is an Open Access article distributed under the terms of the Creative Commons Attribution Non-Commercial License which permits unrestricted non-commercial use, distribution, and reproduction in any medium provided the original work is properly cited 